\section{AUTOCODE: An SAS program to facilitate entry of sparsely coded data}

\section{KENNETH E. FREEDLAND \\ Missouri Institute of Psychiatry, St. Louis, Missouri 63139}

Sparsely coded data sets are characterized by a very large proportion of missing data represented as blank fields. They are produced by dummy and/or "nonsense" coding (Cohen \& Cohen, 1975) of open-ended questionnaire responses, lengthy multiple-response surveys, certain types of behavioral observation protocols, and other applications in which the relevance of individual items varies considerably across cases or sampling periods. The transfer to standard FORTRAN coding sheets and subsequent keypunching of sparse data constitute a difficult, time-consuming, and error-prone process.

AUTOCODE is a brief SAS program designed to eliminate the need for transfer to FORTRAN coding sheets and to improve the speed, ease, and accuracy of keypunching of sparsely coded data. The program's input consists of regularly spaced, densely coded pairs of vectors that are relatively easy to keypunch. The first vector in each pair is a response code, and the second is the column number assigned to that response. The input records also include sequence or identification numbers and card-within-case numbers. The program's output consists of sparsely coded rectangular matrices spanning as many records per case as necessary. These are in the appropriate form for use as raw input by standard statistical packages such as SAS or SPSS. The keypuncher may work directly from the original raw response forms if these include record and column information. Otherwise, responses may be transferred to "codebook" forms (one form per case).

Limitations. In its present form, AUTOCODE will only accept responses that can be represented by a single digit. The program can easily be modified by the user to handle responses coded in double or triple digits. However, the number would have to remain constant across responses in order to maintain the spatial regularity of the input data. Also, the program may be used only at installations that support SAS.

Availability. A listing of the program and a set of instructions may be obtained without charge from Kenneth E. Freedland, Missouri Institute of Psychiatry, 5400 Arsenal Street, St. Louis, Missouri 63139.

\section{REFERENCES}

Cohen, J., \& Cohen, P. Applied multiple regression/correlation analysis for the behavioral sciences. New York: Wiley, 1975. SAS user's guide. Raleigh: SAS Institute, Inc., 1979.

(Accepted for publication October 28, 1982.) 